\title{
New model for MEN1 risk prediction
}

A new model for predicting the risk of multiple endocrine neoplasia type 1 (MEN1) syndrome has been developed by a team in the Netherlands.

"Identifying MEN1 syndrome has important clinical consequences for patients and their families, because of the need of lifelong surveillance to identify manifestations of the disease early," explains senior researcher Gerlof Valk. However, "consensus guidelines on the diagnosis and therapy of MEN1 syndrome were based on limited data," he adds, which led his team to develop and validate a rule to calculate individual risk of MEN1 syndrome.

The researchers carried out a nationwide cross-sectional study in patients who presented with sporadically occurring endocrine tumours and who had clinical characteristics indicating that MEN1 syndrome could be present. Data obtained in 1998-2010 were collected and 365 patients were included in the study, of whom $15.9 \%$ were found to have MEN1 syndrome upon genetic testing.
Recurrent or multiglandular primary hyperparathyroidism was the strongest predictor of MEN1 syndrome (OR 162.40), followed by nonrecurrent primary hyperparathyroidism (OR 25.78), neuroendocrine tumour (NET) of the stomach, thymus or bronchus (OR 25.84), pancreatic or duodenal NETs (OR 17.94), pituitary tumour (OR 4.71), positive family history of NET (OR 4.53) and age (OR 0.96).

The researchers then constructed a nomogram that can be used in clinical practice to predict the risk of MEN1 syndrome in individual patients by inclusion of scores for each risk factor, and externally validated the model in a Swedish cohort of 144 patients.

\section{Lian Evans}

Original article de Laat, J. M. et al. Predicting the risk of Multiple Endocrine Neoplasia type 1 (MEN1) for patients with commonly occurring endocrine tumors. Eur. J. Endocrinol. doi:10.1530/EJE-12-0210 